Reprod. Nutr. Dévelop., 1985, 25 (1 A), 61-74.

\title{
Modifications des conditions d'extraction du lait sous l'effet d'un $\beta$-mimétique (Isoprénaline) administré par voie intrajugulaire pendant la traite mécanique de la vache.
}

\author{
J. BERNABÉ, Marie-José RICORDEL
}

Laboratoire de Recherches sur la traite, I.N.R.A., 65, rue de Saint-Brieuc, 35042 Rennes Cedex, France.

Summary. Changes in the conditions of milk extraction under the effect of a $\beta$-receptor agonist (isoprenaline) administered by intrajugular route during the mechanical milking of cows.

Between days 80 and 105 of lactation, 11 FFPN cows (table 1) received an intrajugular injection of either $0.9 \% \mathrm{NaCl}$ or $0.3 \mathrm{mg}$ of isoprenaline (IPN $=\beta$-receptor agonist). Each of the two treatments was repeated at least 7 times by giving $\mathrm{NaCl}$ and IPN on successive, alternate days.

IPN :

- did not change either the total milk yield of the morning milking ( 13.5 | vs 13.37 I with $\mathrm{NaCl}$ ) or the volume of milk obtained by stripping $(0.460$ I vs 0.447 I with $\mathrm{NaCl}$ ), although individual responses varied considerably (table 3 ) ;

- significantly increased milk flow by about $25 \%$ because it reached $1.144 \mathrm{l} / \mathrm{min} /$ quarter after IPN administration vs $0.929 \mathrm{l} / \mathrm{min} /$ quarter after $\mathrm{NaCl}$ injection (table 4).

These results confirm our previous observations concerning milk loss between milkings (Bernabé and Peeters, 1980), i.e. the stimulation of smooth muscle $\beta$-receptors enhances milk flow.

\section{Introduction.}

Les trayons de vaches en lactation se contractent spontanément peu avant la traite (Peeters et al., 1948 ; Sambraus, 1971) et les essais pharmacologiques conduits chez ce type d'animal soit in vitro (Peeters et al., 1977) soit in vivo (Peeters et de Bruycker, 1975 ; Bernabé et Peeters, 1980) démontrent l'existence de récepteurs adrénergiques de type $\alpha$ et $\beta$ au niveau des muscles lisses de l'organe.

En outre, les vaches, dont la citerne est abondamment remplie de lait, présentent fréquemment des écoulements intermittents de lait consécutifs à des relâchements musculaires des trayons (Bernabé et Peeters, 1980). L'injection d'un $\alpha$ mimétique dans l'artère pudique externe provoque un ralentissement (voire un arrêt) de ces « pertes de lait " alors que celle d'un $\beta$-mimétique se traduit par une 
augmentation significative de celles-ci (Bernabé et Peeters, 1980). Dans l'espèce bovine, la modulation du tonus du sphincter du trayon, par activation des adrénorécepteurs, entraîne donc une modification des quantités de lait émises avant la traite.

II nous est apparu souhaitable de connaître également l'influence des sympathicomimétiques pendant la traite mécanique proprement dite et de vérifier en particulier si l'activation des $\beta$-récepteurs postsynaptiques des muscles lisses des trayons par un $\beta$-mimétique sélectif (l'isoprénaline = IPN) entraîne une augmentation du débit lacté et une modification de la quantité de lait produite.

\section{Matériel et méthodes.}

1) Présentation générale de l'essai. - L'essai, qui porte sur 11 vaches FFPN (voir 2), consiste :

- à injecter au cours de la traite mécanique du matin, légèrement retardée (voir 3), soit une solution de $0,3 \mathrm{mg}$ d'IPN soit une solution témoin de $\mathrm{NaCl} 0,9 \%$; - à contrôler, à l'aide d'un système d'enregistrement automatique (voir 4) les modifications qui en résultent sur le débit du lait ou sur les volumes extraits avant et après l'égouttage à la machine (voir 6).

Les deux solutions sont administrées grâce à un cathéter placé en permanence dans la veine jugulaire (voir 5). Chacune d'entre elles est injectée au minimum 7 fois sur chaque vache $\left({ }^{1}\right)$. Les traitements IPN et témoin ( $\mathrm{NaCl}$ ) sont alternés un jour sur deux, samedi et dimanche exceptés. L'expérience dure donc trois semaines par vache et se situe aux environs du $90^{e}$ jour de la lactation (voi. 2 ).

2) Caractéristiques des animaux et conduite d'élevage. - Les principales caractéristiques zootechniques sont rapportées au tableau 1.

Les animaux sont entretenus en permanence dans une stabulation libre sur paille. Ils reçoivent un régime à base d'ensilage de maîs $18,5 \mathrm{~kg}$ de matière sèche/jour) et de foin de graminées (ad libitum). Du tourteau de soja ( ${ }^{2}$ ) est mélangé à l'ensilage à raison de $1,5 \mathrm{~kg}$ de complément azoté par vache.

L'aliment " concentré " $\left({ }^{3}\right)$ est distribué pendant la traite à raison de 1 $\mathrm{kg}$ /jour par unité de 2,5 litres de lait au-dessus d'une production de base de $15 \mathrm{l} /$ jour. Enfin, une complémentation minérale phosphocalcique est ajoutée en mélange à l'ensilage de maïs (soit $200 \mathrm{~g} /$ jour et par vache).

3) Technique et matériel de traite. - La salle de traite utilisée est composée de 8 stalles « tandem ». Les traites expérimentales, effectuées du lundi au ven$0.9 \%)$.

(1) Les vaches 158, 182 et 189 ont reçu respectivement 18, 20 et 18 injections (IPN $+\mathrm{NaCl}$

(2) Aliment composé complémentaire type "Vache 380 »-Coopérative agricole rennaise $0,92 \mathrm{UFL} / \mathrm{kg}$.

(3) Aliment composé complémentaire type "Vache laitière 185 " - Coopérative agricole rennaise $-0,90 \mathrm{UFL} / \mathrm{kg}$. 
TABLEAU 1

Principales caractéristiques zootechniques des animaux étudiés.

\begin{tabular}{|c|c|c|c|c|c|c|c|}
\hline \multirow{3}{*}{$\begin{array}{l}\text { Vache } \\
\mathrm{N}^{\circ}\end{array}$} & \multirow{3}{*}{$\begin{array}{c}\text { Poids a } \\
\text { J5 (1) } \\
(\mathrm{kg})\end{array}$} & \multirow{3}{*}{$\begin{array}{c}\mathrm{N}^{\circ} \\
\text { lactation }\end{array}$} & \multicolumn{4}{|c|}{ Expérimentation } & \multirow{3}{*}{$\begin{array}{l}\text { Insémination } \\
\text { artificielle } \\
\text { Stade }\left\langle{ }^{1}\right\}\end{array}$} \\
\hline & & & \multicolumn{2}{|c|}{ Début } & \multicolumn{2}{|c|}{ Fin } & \\
\hline & & & Stade (1) & $\begin{array}{l}\text { Lait } \\
\text { (litres/ } \\
\text { jour) }\end{array}$ & Stade (1) & $\begin{array}{c}\text { Lait } \\
\text { (litres/ } \\
\text { jour) }\end{array}$ & \\
\hline 120 & 626 & 6 & 79 & 29,0 & 103 & 18,5 & 83 \\
\hline 135 & 590 & 4 & 13 & 25,4 & 28 & 23,5 & - \\
\hline 136 & 675 & 4 & 80 & 23,0 & 102 & 21,0 & - \\
\hline 146 & 666 & 3 & 72 & 28,0 & 104 & 25,5 & - \\
\hline 153 & 552 & 3 & 101 & 24,5 & 123 & 12,0 & - \\
\hline 158 & 572 & 3 & 91 & 29,0 & 119 & 16,0 & 80 \\
\hline 181 & 532 & 1 & 113 & 21,3 & 137 & 16,0 & 81 \\
\hline 182 & 515 & 1 & 78 & 23,7 & 102 & 17,0 & - \\
\hline 186 & 452 & 1 & 92 & 19,3 & 122 & 13,0 & 72 \\
\hline 187 & 451 & 1 & 97 & 16,0 & 115 & 15,0 & 64 \\
\hline 189 & 470 & 1 & 74 & 21,0 & 98 & 10,0 & - \\
\hline Moyenne & 555 & 2,5 & 81 & 23,7 & 105 & 17,1 & - \\
\hline $\begin{array}{l}\text { Écart- } \\
\text { type }\end{array}$ & 80 & 1,7 & 26 & 4,1 & 28 & 4,8 & - \\
\hline
\end{tabular}

(1) Les stades de pesée, d'însémination et de lactation sont exprimés en jour à partir du vêlage $(\mathrm{J} 0)$.

dredi, ont toujours lieu dans la même stalle entre 9 h et $11 \mathrm{~h} 30\left({ }^{4}\right)$. Les animaux sont traits un par un, dans les conditions suivantes.

Après distribution du concentré, le trayeur pratique un massage manuel énergique (lavette, eau à $40^{\circ} \mathrm{C}$ ) de $30 \mathrm{~s}$. Les gobelets ne sont mis en place que $30 \mathrm{~s}$ plus tard $\left({ }^{5}\right)$ toujours dans le même ordre: postérieur droit (PD), antérieur droit $(A D)$, antérieur gauche $(A G)$ et postérieur gauche $(P G)$. Le temps de pose dure environ $6 \mathrm{~s}$. Lorsque le débit du quartier le plus lent devient inférieur à $10 \mathrm{ml} / \mathrm{min}$ environ, on procède à un égouttage de 20 à $30 \mathrm{~s}$ avant que la machine à traire ne soit retirée des trayons.

(4) Les conditions habituelles de traite sont légèrement modifiées puisque " normalement »: - les horaires vont de $7 \mathrm{~h} 30$ à $8 \mathrm{~h} 30$ et de $16 \mathrm{~h}$ à $17 \mathrm{~h}$; - les animaux s'orientent librement selon leur ordre d'entrée en salle de traite vers l'une des 8 stalles.

(5) Nous souhaitons ainsi éviter une évacuation successive et distincte du lait citernal et alvéolaire et minimiser l'influence du trayeur puisque 4 vachers ont participé à l'expérience. 
Les principales caractéristiques techniques de celle-ci sont:

- niveau de vide, mesuré dans le lactoduc : $50,67 \mathrm{kPa}$;

- rapport de pulsation, enregistré dans la chambre de pulsation : 71,88\% ;

- vitesse de pulsation : $60 \mathrm{C} / \mathrm{min}$;

- gobelets Alfa-Laval $n^{\circ} 25659$;

- manchons trayeurs Alfa-Laval $n^{\circ}$ 960000-03 ;

- griffe expérimentale, sans entrée d'air, permettant la collecte du lait par quartier séparé $\left({ }^{6}\right)$ :

— poids du faisceau : $2800 \mathrm{~g}$.

4) Enregistrement de la cinétique d'émission du lait. - La chaîne de mesure utilisée est dérivée de celle décrite par Labussière et Martinet (1964). Elle est composée de 4 pots trayeurs associés à 4 suiveurs de niveau photoélectriques (type Sefram SN 50, Paris) reliés à 2 tables traçantes potentiométriques double-voie (JJ Lloyd - CR 600) permettant un enregistrement continu de la quantité de lait émise par chaque trayon. Chaque pot-trayeur est constitué d'un cylindre de verre ( $\theta 150 \mathrm{~mm}$ ), haut de $55 \mathrm{~cm}$, dont l'étanchéité est assurée par 2 embases en acier inoxydable. Le volume maximum de lait mesurable pour chaque quartier est de 8,5 litres environ. L'écart de positionnement des suiveurs de niveau par rapport au ménisque de lait n'excède pas $0,2 \mathrm{~mm}$; l'erreur de lecture ne dépasse donc pas $\pm 3,5 \mathrm{ml}$ de lait.

5) Protocole d'injection. - Afin d'écarter toute frayeur et de diminuer les décharges endogènes de catécholamines pendant la traite, les injections du mimétique ou de $\mathrm{NaCl}$ sont effectuées grâce à un cathéter $(30 \mathrm{~cm})$ de polyéthylène stérile (PE 90, volume $=0,3 \mathrm{ml}$, Becton et Dickinson) placé trois jours avant le début de l'essai dans la veine jugulaire. Les jours d'expérience, une longue tubulure de vinyl ( $L=2,5 \mathrm{~m}$; volume $=2 \mathrm{ml}$ ) est interposée entre le cathéter jugulaire et la seringue, pour éloigner l'expérimentateur de l'animal. Le volume d'injection d'IPN ou de $\mathrm{NaCl}$ est toujours de $5 \mathrm{ml}$. II est immédiatement suivi d'un rinçage des cathéters par $3 \mathrm{ml}$ de $\mathrm{NaCl} 0,9 \%$ ce qui assure une pénétration totale de la substance à tester dans le système circulatoire de l'animal. Trois milligrammes d'isoprénaline $( \pm$ isoproterenol $\mathrm{HCl}$; Sigma, réf. 1-5627) sont dissous dans $50 \mathrm{ml}$ d'une solution de $\mathrm{NaCl} 0,9 \%$ et d'acide ascorbique $(0,6 \mu \mathrm{g} / \mathrm{ml})$. Les préparations sont réalisées extemporanément et stérilisées par ultrafiltration (filtre Millipore stérile - pore de $0,2 \mu \mathrm{m}$ ). Elles sont injectées en cours de traite ; le temps séparant l'injection de la pose du dernier gobelet trayeur (PG) varie entre 30 et 150 secondes (tabl. 2). Cette valeur (1/2 t), qui correspond approximativement à la moitié du temps d'écoulement $(\mathrm{t})$ du quartier le plus rapide à traire, permet la comparaison des débits de pré et post-injection (voir figure 1).

(6) L'absence d'entrée d'air peut se traduire par une augmentation du vide sous le trayon et une remontée plus rapide des gobelets en fin de traite. Le phénomène pourrait augmenter l'importance du lait d'égouttage (Le Du et Richard, 1977). 
TABLEAU 2

Emplacement et durée des injections d'IPN et de $\mathrm{NaCl}$ au cour de la traite mécanique.

\begin{tabular}{cccc}
\hline Vaches & $\begin{array}{c}\text { Moment de l'injection } \\
\text { (secondes) (1) }\end{array}$ & $\begin{array}{c}\text { Durée de l'injection } \\
\text { (secondes) (1) }\end{array}$ \\
\hline 120 & 30 & 1 NaCl & 5 \\
\hline 135 & 80 & 12 & 7 \\
\hline 136 & 100 & 15 & 6 \\
\hline 146 & $(2)$ & 15 & 6 \\
\hline 153 & 150 & 13 & 7 \\
\hline 158 & 150 & 15 & 6 \\
\hline 181 & 80 & 15 & 6 \\
\hline 182 & 75 & 15 & 6 \\
\hline 186 & 60 & 16 & 7 \\
\hline 187 & 80 & 15 & 6,2 \\
\hline 189 & 80 & 15 & 6,6 \\
\hline Ecart-Type & 88,5 & 1,8 & 6 \\
\hline
\end{tabular}

(1) Par rapport à la pose du $4^{\mathrm{e}}$ gobelet trayeur. trayeur.

(2) La vache 146 reçoit les injections de $\mathrm{NaCl}$ ou d'IPN 20 secondes avant la pose du for gobelet

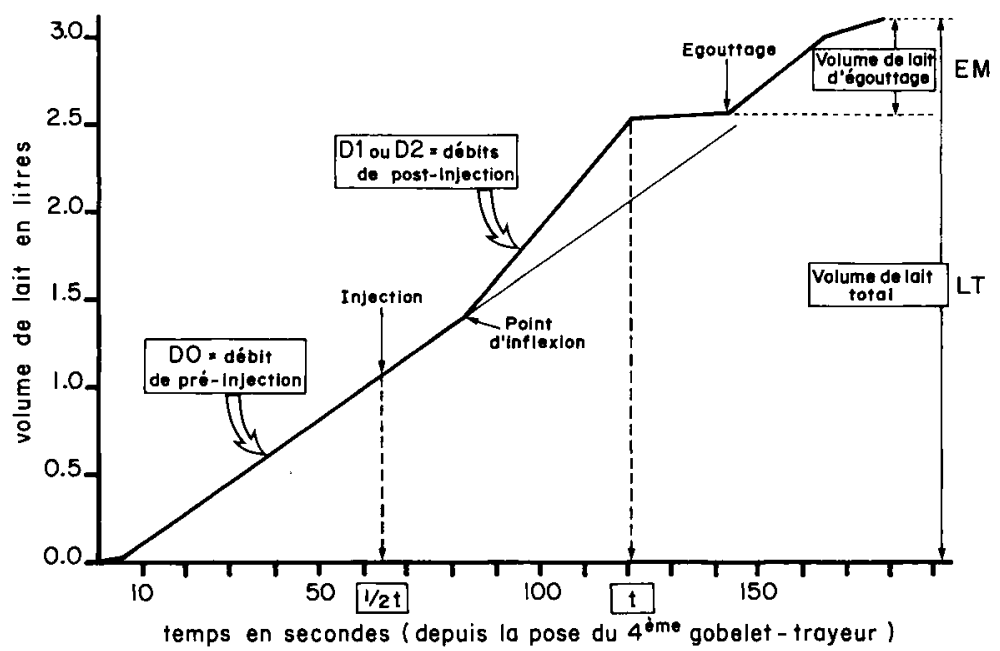

FIG. 1. - Courbe type de la cinétique d'émission du lait d'un quartier et définition des paramètres mesurés.

$\mathbf{t}=$ temps, pour une vache, où le débit du quartier le plus rapide est constant ; $1 / 2 \mathrm{t}=$ correspond au moment de l'injection intraveineuse. 
6) Détermination des débits de lait et paramètres retenus dans l'analyse. Pour chaque quartier et pour chaque traite, quatre paramètres sont calculés à partir des courbes d'enregistrements de la cinétique d'émission du lait, dont un exemple est présenté à la figure 1 . Ces paramètres sont les suivants :

- production totale de lait (PT en I),

- volume du lait d'égouttage (EM en I),

- débit du lait avant injection de $\mathrm{NaCl}$ ou d'IPN (DO en I/min),

- débit du lait après injection de $\mathrm{NaCl}$ (D1 en $1 / \mathrm{min}$ ),

- débit du lait après injection d'IPN (D2 en I/min).

Les volumes de lait produit par quartier sont transformés de millimètres en litres grâce à un étalonnage préalable du système. Les débits de lait sont obtenus par calcul des pentes des droites situées de part et d'autre du point d'inflexion consécutif à l'action du produit. En l'absence d'un tel point (en particulier pour $\mathrm{NaCl}$, nous avons décidé de calculer les pentes en fonction d'un point fictif choisi $30 \mathrm{~s}$ après l'injection puisque ce délai peut être considéré comme suffisant pour assurer le transport de la solution à tester de la veine jugulaire jusqu'à la mamelle ( 7 ).

\section{Résultats.}

Effets de l'isoprénaline sur la production du lait à la traite du matin (PT). La figure 2 indique qu'une administration intrajugulaire de $0,3 \mathrm{mg}$ d'IPN en cours de traite, ne modifie pas la production laitière moyenne des 11 vaches soumises à

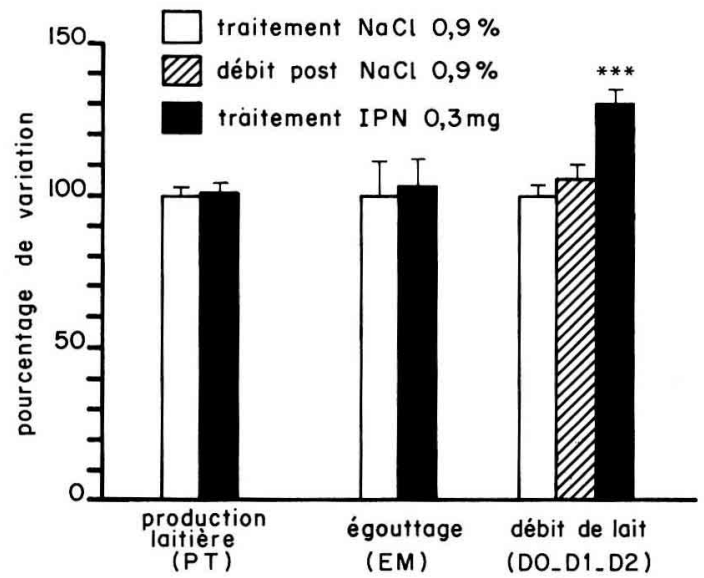

FIG. 2. - Effets de I'IPN sur la production totale de lait (PT) et le volume d'égouttage (EM), les débits lactés (D0, D1 et D2) pendant la traite mécanique.

Les valeurs sont exprimées en pourcentage de variation par rapport aux valeurs obtenues sous traitement $\mathrm{NaCl}(P T, E M)$ ou avant injection de $\mathrm{NaCl}$ (DO). Les lignes verticales représentent l'erreur à la moyenne (SEM).

(7) Ce temps de transport est en effet de 18 à 20 secondes pour l'ocytocine (Vercruisse, Peeters, et Coussens, 1950). 
I'essai $(13,37$ I sous $\mathrm{NaCl}$ témoin contre 13,51 I sous IPN). II apparaît en effet à la figure 3 et au tableau 3 (sans que l'effet intravache soit significatif) que :

- 5 animaux fournissent entre 0,09 । et 1,70 । de lait en plus (soit $+0,8 \%$ à $+10,8 \%)^{(8)}$ alors que les 6 autres produisent, au contraire, un peu moins (entre $-0,06$ I et $-0,73$ I de lait soit une baisse de $-0,5 \%$ à $-4,8 \%$ ). II est à cet égard intéressant de remarquer que, parmi les 5 sujets répondant positivement, 3 d'entre eux (146, 158 et 186) présentent une augmentation de production sur

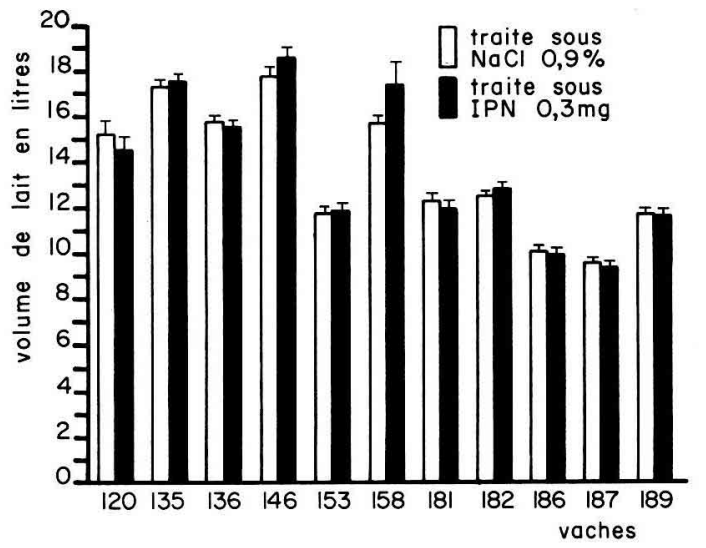

FIG. 3. - Influence de I'IPN sur la production laitière (PT) de chacune des 11 vaches, à la traite du matin $( \pm$ SEM).

TABLEAU 3

Effets des injections de NaCl et d'IPN sur les productions totales de lait (PT) et les volumes d'égouttage (EM) de chaque quartier.

\begin{tabular}{|c|c|c|c|c|c|c|c|}
\hline & & & PT & & & EM & \\
\hline $\begin{array}{c}\text { Vache } \\
N^{\circ}\end{array}$ & Quartier & $\begin{array}{l}\text { Lait total } \\
\text { produit sous } \\
\text { influence de } \\
\mathrm{NaCl} 0,9 \%\end{array}$ & $\begin{array}{l}\text { Lait total } \\
\text { produit sous } \\
\text { influence } \\
\text { d'IPN } 0,3 \mathrm{mg}\end{array}$ & $\begin{array}{l}\text { Effet de } \\
\text { I'IPN } \\
\text { sur le lait } \\
\text { total }\end{array}$ & $\begin{array}{c}\text { Lait } \\
\text { d'égouttage } \\
\text { produit sous } \\
\text { influence de } \\
\mathrm{NaCl}\end{array}$ & $\begin{array}{c}\text { Lait } \\
\text { d'égouttage } \\
\text { produit sous } \\
\text { influence } \\
\text { d'IPN }\end{array}$ & $\begin{array}{c}\text { Effet de } \\
\text { I'IPN } \\
\text { sur le lait } \\
\text { d'égouttage }\end{array}$ \\
\hline 120 & $\begin{array}{l}\text { P.D. } \\
\text { A.D. } \\
\text { A.G. } \\
\text { P.G. } \\
\text { Total }\end{array}$ & $\begin{array}{r}5,275 \\
2,978 \\
2,112 \\
4,913 \\
15,278\end{array}$ & $\begin{array}{r}4,415 \\
3,026 \\
2,184 \\
4,625 \\
14,550\end{array}$ & $\begin{array}{l}-0,560 \\
+0,048 \\
+0,072 \\
-0,288 \\
-0,728\end{array}$ & $\begin{array}{l}0,340 \\
0,037 \\
0,045 \\
0,530 \\
0,955\end{array}$ & $\begin{array}{l}0,360 \\
0,100 \\
0,070 \\
0,670 \\
1,200\end{array}$ & $\begin{array}{l}+0,020 \\
+0,063^{* * *} \\
+0,025^{*} \\
+0,140 \\
+0,245\end{array}$ \\
\hline 135 & $\begin{array}{l}\text { P.D. } \\
\text { A.D. } \\
\text { A.G. } \\
\text { P.G. } \\
\text { Total }\end{array}$ & $\begin{array}{r}6,044 \\
3,648 \\
2,330 \\
5,358 \\
17,380\end{array}$ & $\begin{array}{r}6,189 \\
3,802 \\
2,231 \\
5,385 \\
17,607\end{array}$ & $\begin{array}{l}+0,145 \\
+0,154 \\
-0,099 \\
+0,027 \\
+0,227\end{array}$ & $\begin{array}{l}0,180 \\
0,114 \\
0,035 \\
0,101 \\
0,418\end{array}$ & $\begin{array}{l}0,255 \\
0,070 \\
0,053 \\
0,176 \\
0,557\end{array}$ & $\begin{array}{l}+0,075 \\
-0,044 \\
+0,018 \\
+0,075 \\
+0,139\end{array}$ \\
\hline
\end{tabular}

Effet significatif au seuil de $0,05\left(^{*}\right) 0,01\left(^{* *}\right)$ et $\left.0,0011^{* *}\right)$.

P.D. : postérieur droit ; A.D. : antérieur droit ; A.G. : antérieur gauche ; P.G. : postérieur gauche.

(8) $100 \%=$ production laitière observée lors de l'injection de $\mathrm{NaCl} 0,9 \%$. 
TABLEAU 3 (suite)

\begin{tabular}{|c|c|c|c|c|c|c|c|}
\hline 136 & $\begin{array}{l}\text { P.D. } \\
\text { A.D. } \\
\text { A.G. } \\
\text { P.G. } \\
\text { Total }\end{array}$ & $\begin{array}{r}5,123 \\
3,508 \\
3,054 \\
4,146 \\
15,831\end{array}$ & $\begin{array}{r}5,174 \\
3,622 \\
2,818 \\
3,974 \\
15,588\end{array}$ & $\begin{array}{l}+0,051 \\
+0,114 \\
-0,236 \\
-0,172 \\
-0,243\end{array}$ & $\begin{array}{l}0,442 \\
0,138 \\
0,285 \\
0,546 \\
1,575\end{array}$ & $\begin{array}{l}0,110 \\
0,097 \\
0,163 \\
0,255 \\
0,642\end{array}$ & $\begin{array}{l}-0,332^{* *} \\
-0,041^{*} \\
-0,122 \\
-0,291 \\
-0,933^{*}\end{array}$ \\
\hline 146 & $\begin{array}{l}\text { P.D. } \\
\text { A.D. } \\
\text { A.G. } \\
\text { P.G. } \\
\text { Total }\end{array}$ & $\begin{array}{r}4,554 \\
4,369 \\
4,242 \\
4,629 \\
17,794\end{array}$ & $\begin{array}{r}4,725 \\
4,664 \\
4,448 \\
4,796 \\
18,633\end{array}$ & $\begin{array}{l}+0,171 \\
+0,295 \\
+0,206 \\
+0,167 \\
+0,839\end{array}$ & $\begin{array}{l}0,211 \\
0,202 \\
0,790 \\
0,145 \\
0,665\end{array}$ & $\begin{array}{l}0,334 \\
0,255 \\
0,105 \\
0,290 \\
1,011\end{array}$ & $\begin{array}{l}+0,123 \\
+0,053 \\
+0,026 \\
+0,145 \\
+0,346\end{array}$ \\
\hline 153 & $\begin{array}{l}\text { P.D. } \\
\text { A.D. } \\
\text { A.G. } \\
\text { P.G. } \\
\text { Total }\end{array}$ & $\begin{array}{r}3,216 \\
2,673 \\
2,443 \\
3,454 \\
11,786\end{array}$ & $\begin{array}{r}3,323 \\
2,774 \\
2,420 \\
3,357 \\
11,874\end{array}$ & $\begin{array}{l}+0,107 \\
+0,101 \\
-0,023 \\
-0,097 \\
+0,088\end{array}$ & $\begin{array}{l}0,045 \\
0,066 \\
0,021 \\
0,220 \\
0,363\end{array}$ & $\begin{array}{l}0,058 \\
0,076 \\
0,027 \\
0,170 \\
0,341\end{array}$ & $\begin{array}{l}+0,013 \\
+0,010 \\
+0,006 \\
-0,050 \\
-0,022\end{array}$ \\
\hline 158 & $\begin{array}{l}\text { P.D. } \\
\text { A.D. } \\
\text { A.G. } \\
\text { P.G. } \\
\text { Total }\end{array}$ & $\begin{array}{r}6,992 \\
3,316 \\
0,960 \\
4,472 \\
15,740\end{array}$ & $\begin{array}{r}7,617 \\
3,663 \\
1,264 \\
4,894 \\
17,438\end{array}$ & $\begin{array}{l}+0,625 \\
+0,347 \\
+0,304 \\
+0,422 \\
+1,698\end{array}$ & $\begin{array}{l}0,237 \\
0,043 \\
0,265 \\
0,172 \\
0,717\end{array}$ & $\begin{array}{l}0,151 \\
0,091 \\
0,318 \\
0,185 \\
0,745\end{array}$ & $\begin{array}{l}-0,086 \\
+0,048 \\
+0,053 \\
+0,013 \\
+0,028\end{array}$ \\
\hline 181 & $\begin{array}{l}\text { P.D. } \\
\text { A.D. } \\
\text { A.G. } \\
\text { P.G. } \\
\text { Total }\end{array}$ & $\begin{array}{r}3,939 \\
2,662 \\
2,692 \\
3,039 \\
12,332\end{array}$ & $\begin{array}{r}3,877 \\
2,641 \\
2,574 \\
2,895 \\
11,987\end{array}$ & $\begin{array}{l}-0,062 \\
-0,021 \\
-0,118 \\
-0,144 \\
-0,345\end{array}$ & $\begin{array}{c}0,092 \\
0,015 \\
0,009 \\
0 \\
0,116\end{array}$ & $\begin{array}{c}0,034 \\
0,026 \\
0,021 \\
0 \\
0,074\end{array}$ & $\begin{array}{c}-0,058 \\
+0,011 \\
+0,012 \\
0 \\
-0,036\end{array}$ \\
\hline 182 & $\begin{array}{l}\text { P.D. } \\
\text { A.D. } \\
\text { A.G. } \\
\text { P.G. } \\
\text { Total }\end{array}$ & $\begin{array}{r}3,888 \\
2,562 \\
2,613 \\
3,462 \\
12,525\end{array}$ & $\begin{array}{r}3,888 \\
2,580 \\
2,670 \\
3,730 \\
12,868\end{array}$ & $\begin{array}{cl} & 0 \\
+ & 0,018 \\
+ & 0,057 \\
+ & 0,268 \\
+ & 0,343\end{array}$ & $\begin{array}{l}0,038 \\
0,015 \\
0,013 \\
0,024 \\
0,091\end{array}$ & $\begin{array}{l}0,046 \\
0,018 \\
0,023 \\
0,030 \\
0,110\end{array}$ & $\begin{array}{l}+0,008 \\
+0,003 \\
+0,010^{*} \\
+0,006 \\
+0,019\end{array}$ \\
\hline 186 & $\begin{array}{l}\text { P.D. } \\
\text { A.D. } \\
\text { A.G. } \\
\text { P.G. } \\
\text { Total }\end{array}$ & $\begin{array}{r}2,820 \\
2,373 \\
2,027 \\
2,900 \\
10,120\end{array}$ & $\begin{array}{l}2,860 \\
2,376 \\
2,054 \\
2,683 \\
9,973\end{array}$ & $\begin{array}{l}+0,040 \\
+0,003 \\
+0,027 \\
-0,217 \\
+0,147\end{array}$ & $\begin{array}{l}0,108 \\
0,017 \\
0,024 \\
0,190 \\
0,312\end{array}$ & $\begin{array}{l}0,097 \\
0,042 \\
0,033 \\
0,180 \\
0,361\end{array}$ & $\begin{array}{l}-0,011 \\
+0,025^{*} \\
+0,009 \\
-0,010 \\
+0,049\end{array}$ \\
\hline 187 & $\begin{array}{l}\text { P.D. } \\
\text { A.D. } \\
\text { A.G. } \\
\text { P.G. } \\
\text { Total }\end{array}$ & $\begin{array}{l}2,949 \\
1,872 \\
1,829 \\
2,963 \\
9,613\end{array}$ & $\begin{array}{l}2,920 \\
1,833 \\
1,805 \\
2,840 \\
9,398\end{array}$ & $\begin{array}{l}-0,029 \\
-0,039 \\
-0,024 \\
-0,123 \\
-0,215\end{array}$ & $\begin{array}{l}0,033 \\
0,026 \\
0,015 \\
0,021 \\
0,092\end{array}$ & $\begin{array}{l}0,052 \\
0,024 \\
0,018 \\
0,040 \\
0,149\end{array}$ & $\begin{array}{l}+0,190^{*} \\
-0,002 \\
+0,003 \\
+0,019 \\
+0,057\end{array}$ \\
\hline 189 & $\begin{array}{l}\text { P.D. } \\
\text { A.D. } \\
\text { A.G. } \\
\text { P.G. } \\
\text { Total }\end{array}$ & $\begin{array}{r}2,407 \\
3,124 \\
2,955 \\
3,265 \\
11,751\end{array}$ & $\begin{array}{r}2,379 \\
3,135 \\
2,938 \\
3,237 \\
11,689\end{array}$ & $\begin{array}{l}-0,028 \\
+0,011 \\
-0,017 \\
-0,028 \\
-0,062\end{array}$ & $\begin{array}{l}0,036 \\
0,012 \\
0,026 \\
0,018 \\
0,094\end{array}$ & $\begin{array}{l}0,044 \\
0,019 \\
0,035 \\
0,023 \\
0,111\end{array}$ & $\begin{array}{l}+0,008 \\
+0,007 \\
+0,009 \\
+0,005 \\
+0,017\end{array}$ \\
\hline \multicolumn{2}{|c|}{$\begin{array}{l}\text { Moyenne } \\
\text { générale }\end{array}$} & 13,369 & 13,507 & $+0,130$ & 0,447 & 0,460 & $+0,013$ \\
\hline \multicolumn{2}{|c|}{ SEM } & 0,315 & 0,348 & 0,068 & 0,051 & 0,043 & 0,036 \\
\hline
\end{tabular}

Effet significatif au seuil de $0,05\left({ }^{*}\right) 0,01\left(^{* *}\right)$ et $0,001(* *)$.

P.D. : postérieur droit ; A.D. : antérieur droit ; A.G. : antérieur gauche ; P.G. : postérieur gauche. 
chacun des 4 trayons. Cette homogénéité de réponse des quartiers est également observée sur 3 des 6 vaches $(181,187$ et 189) dont la production semble réduite après IPN.

- une répartition strictement égale des quartiers dont la production est légèrement réduite (22) ou accrue (22) sans qu'il soit possible de relier l'augmentation ou la baisse à la position antérieure ou postérieure des trayons.

Influence de I'IPN sur le volume de lait obtenu lors de l'égouttage à la machine (EM). - Si l'on considère l'ensemble des 11 vaches soumises à cette étude, il ressort de l'examen de la figure 2 que I'IPN ne modifie pas le volume d'égouttage recueilli à la machine avant la dépose des gobelets $(0,447$ I sous $\mathrm{NaCl}$ témoin contre $0,460 \mathrm{I}$ sous IPN). Ces valeurs moyennes très voisines cachent toutefois des variations individuelles de réponses à l'IPN très importantes (tabl. 3). C'est ainsi que l'animal 136 présente un égouttage significativement plus faible ( - $59 \%$ ) sous l'effet de I'IPN alors qu'au contraire la vache 146 voit le sien accru de $50 \%$. Cette tendance à l'augmentation est d'ailleurs constatée dans la majorité des cas ( 7 vaches sur 11,32 quartiers sur 44 dont 5 cas significatifs) et semble plus nette chez les multipares qui ont un plus fort volume d'égouttage.

Effets de l'isoprénaline sur le débit d'émission du lait.

- Pendant la première moitié de la traite et avant toute injection, le débit moyen (DO) calculé sur 10 vaches $\left({ }^{9}\right)$ est de $0,880 \mathrm{I} / \mathrm{min}$. La vache 120 présente la valeur la plus élevée $(1,58 \mathrm{I} / \mathrm{min})$ alors que les animaux 136 et 153 donnent leur lait 3 fois moins rapidement (environ $0,5 \mathrm{l} / \mathrm{min}$ ) (fig. 4).

- Après injection de $\mathrm{NaCl} 0,9 \%$, la moyenne des débits (D1) passe à $0,929 \mathrm{l} / \mathrm{min}$ soit une légère augmentation de $+5,5 \%$ et touche pratiquement

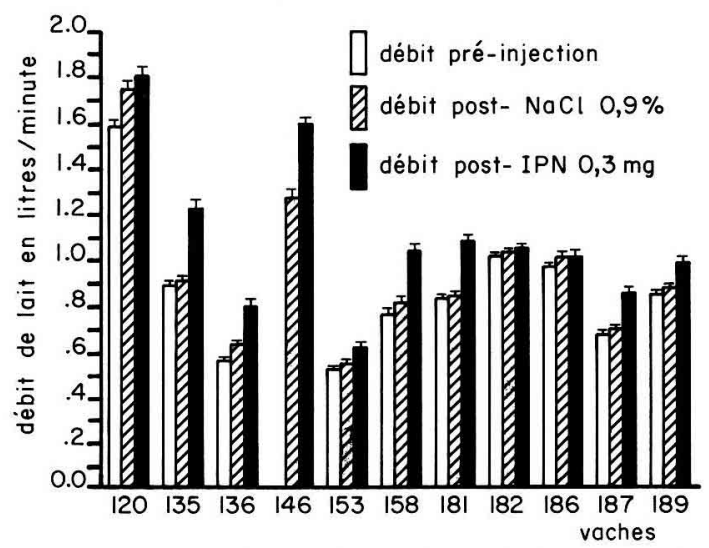

FIG. 4. - Influence des traitements IPN et $\mathrm{NaCl}$ sur le débit du lait de chaque vache pendant la traite mécanique $(+\mathrm{SEM})$

débit moyen de pré-injection (D0) ; $\mathbb{Z}$ débit moyen suivant l'injection de $\mathrm{NaCl}$ (D1) ; débit moyen suivant l'injection d'IPN (D2).

(9) Les données concernant la vache 146 ne peuvent entrer en ligne de compte pour ce calcul puisque les injections d'IPN ont été réalisées avant la traite. 
tous les animaux : les valeurs extrêmes vont de $0 \%$ à $+17 \%$ mais ne sont significatives que pour les vaches $120,136,186$ et 187 .

- L'injection d'IPN (0,3 mg) se traduit (par rapport à D1) par une augmentation significative (fig. 4) du débit D2 de $+24,5 \%$ (extrêmes $+5 \%$ et $+45 \%$ ). Le lait s'écoule alors à $1,144 \mathrm{l} / \mathrm{min}$. L'effet est visible sur les courbes d'enregistrements : un point d'inflexion apparaît moins de $20 \mathrm{~s}$ après la fin de l'injection d'IPN (fig. 5).

Seuls, les animaux 120 et 186 ne semblent pas réagir à I'IPN plus qu'à la solution de $\mathrm{NaCl}$. Les résultats pour les 44 quartiers, fournis au tableau 4 , font

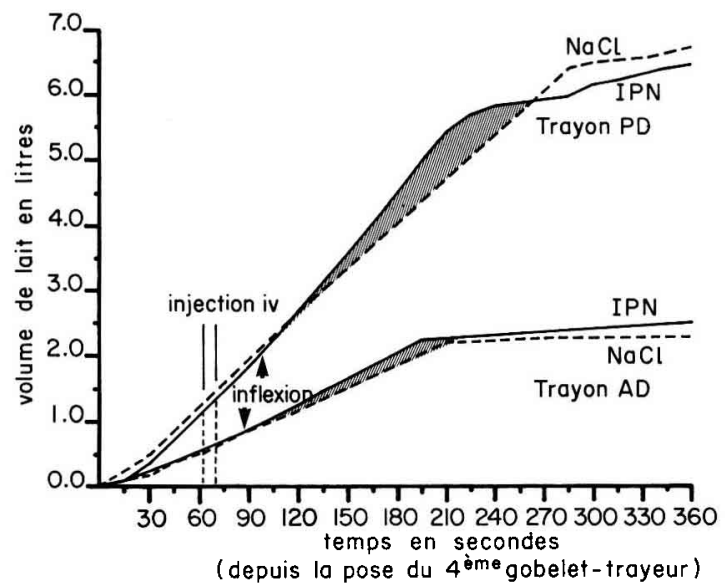

FIG. 5. - Courbes d'émission du lait chez la vache 135: Effets de I'lPN sur le débit d'écoulement

$\|$ : début et fin de l'injection intraveineuse (iv) ;

$Y$ : localisation du point d'inflexion après traitement IPN.

La zone grisée souligne l'effet de I'IPN.

TABLEAU 4

Effets des injections de $\mathrm{NaCl}$ et d'lPN sur le débit lacté de chaque quartier.

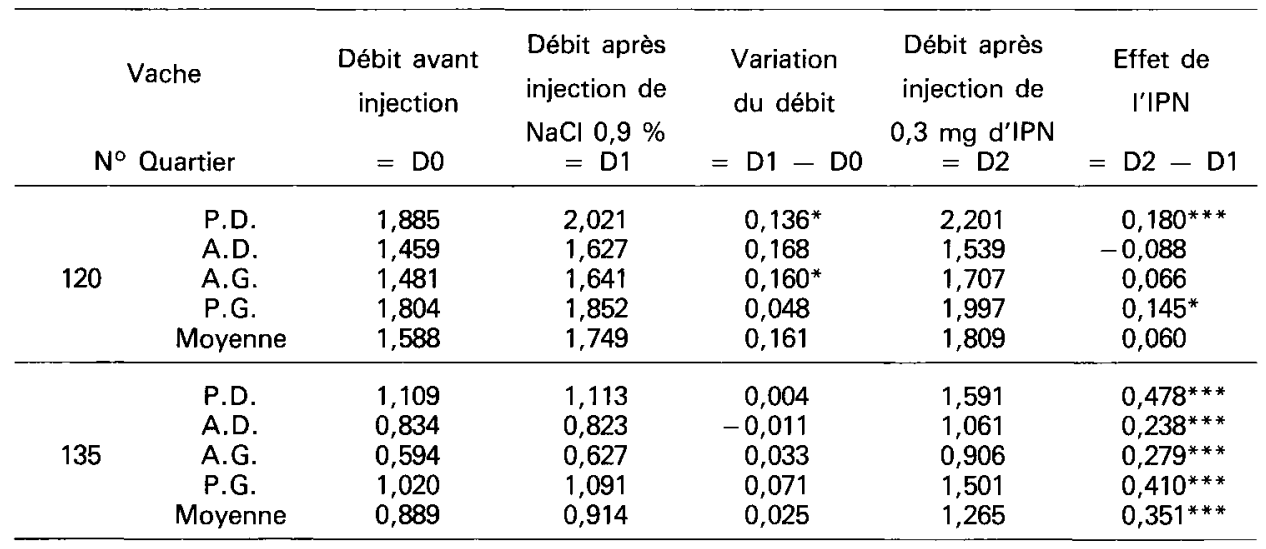

D0 : débit d'écoulement du lait avant toute injection ; D1 : débit d'écoulement du lait après injection de $\mathrm{NaCl} 0,9 \%$; $\mathrm{D} 2$ : débit d'écoulement du lait après injection d'IPN 0,3 mg.

Effet significatif au seuil de $0,05\left(^{*}\right) ; 0,01\left(^{* *}\right)$ et $0,001\left(^{* *}\right)$. 
TABLEAU 4 (suite)

\begin{tabular}{|c|c|c|c|c|c|c|}
\hline 136 & $\begin{array}{l}\text { P.D. } \\
\text { A.D. } \\
\text { A.G. } \\
\text { P.G. } \\
\text { Moyenne }\end{array}$ & $\begin{array}{l}0,610 \\
0,592 \\
0,684 \\
0,400 \\
0,558\end{array}$ & $\begin{array}{l}0,665 \\
0,631 \\
0,821 \\
0,498 \\
0,653\end{array}$ & $\begin{array}{l}0,055 \\
0,039 \\
0,137^{* * *} \\
0,098^{* * *} \\
0,095\end{array}$ & $\begin{array}{l}0,981 \\
0,753 \\
0,811 \\
0,707 \\
0,813\end{array}$ & $\begin{array}{l}0,316^{* * *} \\
0,122^{*} \\
-0,010 \\
0,209^{* * *} \\
0,160^{* * *}\end{array}$ \\
\hline $146(1)$ & $\begin{array}{c}\text { P.D. } \\
\text { A.D. } \\
\text { A.G. } \\
\text { P.G. } \\
\text { Moyenne }\end{array}$ & & $\begin{array}{l}1,416 \\
1,143 \\
1,249 \\
1,436 \\
1,275\end{array}$ & & $\begin{array}{l}1,883 \\
1,321 \\
1,429 \\
1,913 \\
1,601\end{array}$ & $\begin{array}{l}0,467^{* * *} \\
0,178^{* * *} \\
0,180^{* * *} \\
0,477^{* * *} \\
0,326^{* * *}\end{array}$ \\
\hline 153 & $\begin{array}{c}\text { P.D. } \\
\text { A.D. } \\
\text { A.G. } \\
\text { P.G. } \\
\text { Moyenne }\end{array}$ & $\begin{array}{l}0,569 \\
0,437 \\
0,535 \\
0,558 \\
0,522\end{array}$ & $\begin{array}{l}0,622 \\
0,438 \\
0,569 \\
0,589 \\
0,553\end{array}$ & $\begin{array}{l}0,053 \\
0,001 \\
0,034 \\
0,031 \\
0,031\end{array}$ & $\begin{array}{l}0,665 \\
0,652 \\
0,567 \\
0,613 \\
0,631\end{array}$ & $\begin{array}{c}0,043^{* * *} \\
0,214^{* * *} \\
-0,002 \\
0,024 \\
0,078^{*}\end{array}$ \\
\hline 158 & $\begin{array}{l}\text { P.D. } \\
\text { A.D. } \\
\text { A.G. } \\
\text { P.G. } \\
\text { Moyenne }\end{array}$ & $\begin{array}{l}1,196 \\
0,892 \\
0,109 \\
0,972 \\
0,765\end{array}$ & $\begin{array}{l}1,234 \\
1,023 \\
0,142 \\
1,029 \\
0,815\end{array}$ & $\begin{array}{l}0,038 \\
0,131^{*} \\
0,033^{*} \\
0,057 \\
0,050\end{array}$ & $\begin{array}{l}1,431 \\
1,056 \\
0,242 \\
1,254 \\
1,038\end{array}$ & $\begin{array}{l}0,197^{* * *} \\
0,033 \\
0,100^{* * *} \\
0,225^{* * *} \\
0,223^{* * *}\end{array}$ \\
\hline 181 & $\begin{array}{c}\text { P.D. } \\
\text { A.D. } \\
\text { A.G. } \\
\text { P.G. } \\
\text { Moyenne }\end{array}$ & $\begin{array}{l}0,895 \\
0,752 \\
0,792 \\
0,914 \\
0,833\end{array}$ & $\begin{array}{l}0,956 \\
0,738 \\
0,789 \\
0,906 \\
0,844\end{array}$ & $\begin{array}{r}0,061 \\
-0,014 \\
-0,003 \\
-0,008 \\
0,011\end{array}$ & $\begin{array}{l}1,239 \\
0,997 \\
0,981 \\
1,162 \\
1,085\end{array}$ & $\begin{array}{l}0,283^{* * *} \\
0,259^{* * *} \\
0,192^{* * *} \\
0,256^{* * *} \\
0,241^{* * *}\end{array}$ \\
\hline 182 & $\begin{array}{c}\text { P.D. } \\
\text { A.D. } \\
\text { A.G. } \\
\text { P.G. } \\
\text { Moyenne }\end{array}$ & $\begin{array}{l}0,982 \\
0,972 \\
1,074 \\
0,998 \\
1,005\end{array}$ & $\begin{array}{l}1,004 \\
0,967 \\
1,124 \\
1,032 \\
1,031\end{array}$ & $\begin{array}{r}0,022 \\
-0,005 \\
0,050 \\
0,034 \\
0,026\end{array}$ & $\begin{array}{l}1,068 \\
1,033 \\
1,104 \\
1,004 \\
1,053\end{array}$ & $\begin{array}{c}0,064^{*} \\
0,066^{*} \\
-0,020 \\
-0,028 \\
0,022^{*}\end{array}$ \\
\hline 186 & $\begin{array}{l}\text { P.D. } \\
\text { A.D. } \\
\text { A.G. } \\
\text { P.G. } \\
\text { Moyenne }\end{array}$ & $\begin{array}{l}0,907 \\
0,914 \\
0,957 \\
1,102 \\
0,965\end{array}$ & $\begin{array}{l}0,959 \\
1,007 \\
0,958 \\
1,125 \\
1,012\end{array}$ & $\begin{array}{l}0,052 \\
0,093 \\
0,001 \\
0,023 \\
0,047^{*}\end{array}$ & $\begin{array}{l}0,955 \\
0,939 \\
0,982 \\
1,176 \\
0,995\end{array}$ & $\begin{array}{r}-0,004 \\
-0,068 \\
0,024 \\
0,051 \\
-0,017\end{array}$ \\
\hline 187 & $\begin{array}{l}\text { P.D. } \\
\text { A.D. } \\
\text { A.G. } \\
\text { P.G. } \\
\text { Moyenne }\end{array}$ & $\begin{array}{l}0,702 \\
0,593 \\
0,638 \\
0,698 \\
0,659\end{array}$ & $\begin{array}{l}0,689 \\
0,668 \\
0,719 \\
0,704 \\
0,696\end{array}$ & $\begin{array}{c}-0,013^{*} \\
0,075^{*} \\
0,081^{* * *} \\
0,006 \\
0,037^{*}\end{array}$ & $\begin{array}{l}0,927 \\
0,748 \\
0,789 \\
0,959 \\
0,855\end{array}$ & $\begin{array}{l}0,238^{* * *} \\
0,080^{* * *} \\
0,070^{* * *} \\
0,255^{* * *} \\
0,159 * *\end{array}$ \\
\hline 189 & $\begin{array}{l}\text { P.D. } \\
\text { A.D. } \\
\text { A.G. } \\
\text { P.G. } \\
\text { Moyenne }\end{array}$ & $\begin{array}{l}0,823 \\
0,854 \\
0,736 \\
0,996 \\
0,849\end{array}$ & $\begin{array}{l}0,859 \\
0,889 \\
0,749 \\
1,011 \\
0,877\end{array}$ & $\begin{array}{l}0,036 \\
0,035 \\
0,013 \\
0,015 \\
0,028\end{array}$ & $\begin{array}{l}0,927 \\
1,129 \\
1,088 \\
1,199 \\
0,989\end{array}$ & $\begin{array}{l}0,068^{* * *} \\
0,240^{* * *} \\
0,339^{* * *} \\
0,188^{*} \\
0,112^{*}\end{array}$ \\
\hline \multicolumn{2}{|c|}{ Moyenne générale } & 0,880 & 0,929 & $+0,049$ & 1,144 & $+0,215^{* * *}$ \\
\hline & & 0,033 & 0,045 & 0,006 & 0,038 & 0,011 \\
\hline
\end{tabular}

D0 : débit d'écoulement du lait avant toute injection ; D1 : débit d'écoulement du lait après injection de $\mathrm{NaCl} 0,9 \%$; D2 : débit d'écoulement du lait après injection d'IPN $0,3 \mathrm{mg}$.

Effet significatif au seuil de $0,05\left({ }^{*}\right) ; 0,01\left(^{* *}\right)$ et $0,001\left(^{* *}\right)$.

(1) Chez la vache 146, les injections ont été effectuées avant la traite. 
ressortir une hausse significative du débit pour 31 d'entre eux (26 au seuil de $1 \%$, 5 au seuil de $5 \%$ ).

II est intéressant de remarquer que sur les 13 quartiers dont le débit n'est pas affecté par I'IPN, 8 sont situés à l'avant de la mamelle et que 4 concernent la vache 186 précédemment citée.

\section{Discussion.}

L'isoprénaline ( $\beta$-mimétique sélectif) entraîne chez la chèvre une vasodilatation générale (accompagnée d'une tachycardie avec augmentation significative du volume de l'ondée systolique ; Houvenaghel, 1971) et chez la vache un accroissement du débit artériel mammaire (Dhondt et al., 1973). Ces modifications ne peuvent que faciliter l'arrivée de l'ocytocine vers les cellules myoépithéliales mammaires.

Dans la mesure où l'IPN n'entraîne pas d'augmentation de la production, on peut supposer que :

- dans les conditions habituelles de traite (sans IPN), les quantités d'ocytocine qui atteignent la mamelle sont déjà suffisantes pour provoquer une éjection " complète " du lait alvéolaire. II importe de rappeler à cet égard que des vaches " au calme » et soumises à des injections intraveineuses d'octytocine à des doses croissantes (de 0,02 à $0,30 \mathrm{UI}$ ) produisent toujours la même quantité de lait par traite (Sagi et Gorewit, 1980) ;

- il n'y a pas d'interaction négative entre I'IPN et l'ocytocine pour la stimulation des cellules myoépithéliales. On ne peut néanmoins en conclure qu'il n'existe pas chez la vache de récepteurs $\beta$-adrénergiques au niveau du myoépithélium comme cela a été démontré par Vorherr (1971) chez la ratte et la lapine.

Le lait recueilli lors des opérations d'égouttage peut provenir de la lumière des acini et des sites de stockage distribués au-dessus de l'étranglement généralement constaté en fin de traite au niveau du pli annulaire de la base du trayon. Compte tenu de l'efficacité, précédemment jugée bonne, du réflexe d'éjection du lait, on peut alors estimer que, dans nos conditions expérimentales, l'origine citernale du lait d'égouttage est la plus probable. Comme le volume de celui-ci n'est pas modifié par l'injection d'IPN, il faut probablement admettre que les modifications morphologiques induites par ce $\beta$-mimétique (allongement du trayon, Bernabé et Peeters, 1980) ont peu d'effet sur la rétention mécanique et physique du lait dans les citernes. Cette hypothèse semble effectivement corroborée par les travaux de Mein, Thiel et Clough (1973) qui démontrent clairement qu'une extension du trayon pouvant atteindre $+33 \%$ à $+50 \%$ de la longueur initiale, n'affecte en rien la quantité d'égouttage comme le prédit d'ailleurs le modèle mathématique proposé par Balthazar et Scott en 1978.

Bien que I'IPN influence peu les volumes de lait extraits par la machine, il n'en est pas de même en ce qui concerne l'efficacité de ce produit sur la vitesse d'écoulement du lait. On connaît la dépendance du débit de traite vis-à-vis de la section du canal du trayon (Baxter et al., 1950) et plus précisément de son aptitude à s'ouvrir depuis qu'Andreae (1958) et Labussière (1972) ont pu montrer la parfaite relation existant entre le temps d'écoulement du lait et l'extensibilité des 
tissus de l'extrémité du trayon. Cette disposition du méat à la dilatation résulte à la fois :

- des caractéristiques anatomiques (qualité et quantité des tissus; Kuhne, 1950 ) et physiques de I'organe (Naude et Smith, 1964 ; Notsuki et Goto, 1967 ; Ovesen, 1972 ; Balthazar et Scott, 1978) ;

- du contrôle physiologique des muscles pariétaux et sphinctériens qui s'exerce vraisemblablement par l'intermédiaire du système nerveux sympathique (Peeters et al., 1949 ; Lefcourt, 1982) comme semble le prouver l'augmentation significative du débit de traite après anesthésie soit des trayons (de Buhr, 1958 et Barczynski, 1960), soit du nerf inguinal (Emerson, 1929) de la vache ou après dénervation de la glande mammaire de brebis (Labussière, Martinet et Denamur, 1969).

Au travers de mécanismes d'action différents, l'IPN a donc un effet analogue à l'anesthésie locale ou à la dénervation et aussi bien avant (Bernabé et Peeters, 1980 ) que pendant la traite, ce produit semble agir préférentiellement sur les muscles lisses du trayon (pour faciliter leur relaxation) plutôt que sur les nombreuses anastomoses artérioveineuses qui (en favorisant l'apparition de stases sanguines) auraient dû ralentir le débit du lait.

En conclusion, nos résultats sont en accord avec ceux obtenus par Hamann (1981). Les $\beta$-sympathomimétiques ne semblent pas intervenir sur le tissu myoépithélial et l'expulsion du lait hors des acini-mammaires alors que leur rôle principal serait de relaxer les fibres lisses du trayon pour faciliter l'écoulement du lait citernal.

Reçu en mars 1984.

Accepté en septembre 1984.

Remerciements. - Nous tenons à remercier J. Labussière pour ses conseils, Messieurs F. A. de la Chevalerie, Y. Gauchet, C. Hiard, J. Lebail, E. Legendre, E. Lescouet pour leur assistance technique et Mme Annie Briand pour la dactylographie du manuscrit.

\section{Références}

ANDREAE U., 1958. Messvugen am Zitzenkanal von Kühen zur Ermittlung der Melkbarkeit. Z. Tierz. Züchtbiol., 71, 289-298.

BALTHAZAR J. M., SCOTT N. R., 1978. Deformations of the dairy cow's teat by finite element analysis. Proc. int. Sympos. on Machine Milking, Cincinnati, Ohio (U.S.A.), 63-79.

BARCZYNSKI G., 1960. Ein experimenteller Beitrag zu den anatomisch-funktionellen Grundlagen der Melkeigenschaften des Rindes. Inaug. Diss. Vet. Hannover.

BAXTER E. S., CLARKE P. M., DODD F. H., FOOT A. S., 1950 . Factors affecting the rate of machine milking. J. Dairy Res., 17, 117-127.

BERNABÉ J., PEETERS G., 1980. Studies on the motility of smooth muscles of the teats in lactating cows. J. Dairy Res., 47, 259-275.

BUHR (de) W., 1958. Der Einfluss einer Anaesthesie des Strichkanals und der Zisterne auf die Me/kgeschwindigkeit und aut den Milchdruck. Inaug. Diss. Vet. Hannover.

DHONDT G., HOUVENAGHEL A., PEETERS G., VERSCHOOTEN F., 1973. Influence of vasoactive hormones on mammary blood flow in lactating cows. Arch. int. Pharmacodyn. Ther., 204, 89 . 
EMERSON M. A., 1929. Some observations on the innervation of the udder and teats of the cow and their relation to milk production. J. amer. vet. med. Ass., 74, 372-375.

HAMANN J., 1981. Zum Einfluss einer $\beta$-mimetisch wirksamen Substanz (Planipart) auf das Melkverhalten von Köhen. Tierärztl. Umsch., 36, 237-290.

HOUVENAGHEL A., 1971. Measurements of mammary blood flow in ruminants. Vlaams Diergenesk. Tijdschr., 41, 135-141.

KUHNE M., 1950. Struktorverhältriss im Bereich des Zitzenstrichkanales und ihre Beziehungen zur Melkbarkeit. Inaug. Vet. Diss. Hannover.

LABUSSIĖRE J., 1972. Extensiomètre du trayon. Brevet Français $n^{\circ} 2,102-475$.

LABUSSIEERE J., MARTINET J., 1964. Description de deux appareils permettant le contrôle automatique des débits de lait au cours de la traite à la machine. Premiers résultats obtenus chez la brebis. Ann. Zootech., 13, 199-212.

LABUSSIEERE J., MARTINET J., DENAMUR R., 1969. The influence of the milk ejection reflex on the flow rate during the milking of ewes. J. Dairy Res., 36, 191-201.

LE DU J., RICHARD J., 1977. Influence du manchon trayeur sur les caractéristiques de traite des vaches de race Française Frisonne Pie Noire. Ann. Zootech., 26, 503-512.

LEFCOURT A., 1982. Effect of teat stimulation on sympathetic tone in bovine mammary gland. J. Dairy Sci., 65, 2317-2322.

MEIN G. A., THIEL C. C., CLOUGH P. A., 1973. The patterns of milk flowrate and teat movement in the teatcup liner during milking. Aust. J. Dairy Tech., 28, 26-30.

NAUDE R. T., SMITH A., 1964. The relationship between teat conformation and milkability of the cow. Proc. S. Afr. Soc. anim. Prod., 101-103 (en africans).

NOTSUKI I., GOTO T., 1967. Studies in milkability of dairy cows in machine milking. Relationship between characters of teat and milkability in dairy cows. Bull. nat. Inst. Ind. Chiba, 13, 49-58 (en japonais).

OVESEN E., 1972. Milking ability in relation to size and shape of teats. Anim. Prod., 15, 251-257.

PEETERS G., MASSART L., OYAERT W., COUSSENS R., 1948. Plethysmography applied to the teats of the mammary gland. Arch. int. Pharmacodyn. Ther., 75, 243-255.

PEETERS G., COUSSENS R., SIERENS G., 1949. Physiology of the nerves in the bovine mammary gland. Arch. int. Pharmacodyn. Ther., 79, 62-75.

PEETERS G., de BRUYCKER R., 1975. Influence of sympathomimetic drogs on the motility of bovine teat muscles. J. Dairy Res., 42, 11-19.

PEETERS G., PETRE P., QUINTLIER W., 1977. Nature of adrenoceptors sites in bovine teat muscles. Naunyn-Schmiedeberg's Arch. Pharmacol., 296, 111-115.

SAGI R., GOREWIT R. C., 1980 . Effects of various doses of exogenous oxytocin on milk ejection in cows. J. Dairy Sci., 63-1, 80-81.

SAMBRAUS H. H., 1971. Rhythmische Kontractionen der Rinderzitze. Zbl. Vet. Med. A., 18, 335-340.

VERCRUISSE R., PEETERS G., COUSSENS R., 1950. Circulation time of blood in the lactating cow. Arch. int. Pharmacodyn. Ther., 82, 93.

VORHERR H., 1971. Catecholamine antagonism to oxytocin induced milk ejection. Acta endocr., Suppl. 154, 5-38. 